\title{
Simultaneous Stimulation of Slow-wave Sleep and Growth Hormone Secretion by Gamma-hydroxybutyrate in Normal Young Men
}

\author{
Eve Van Cauter, ${ }^{\star}$ Laurence Plat, ${ }^{\star \S}$ Martin B. Scharf, ${ }^{\ddagger}$ Rachel Leproult, ${ }^{\star}$ Sonya Cespedes, ${ }^{\star}$ Mireille L’Hermite-Balériaux, ${ }^{\S}$ \\ and Georges Copinschis \\ *Department of Medicine, University of Chicago, Illinois 60637; ${ }^{\ddagger}$ Center for Research in Sleep Disorders, Cincinnati, Ohio 45246; and \\ ${ }^{\S}$ Center for the Study of Biological Rhythms (CERB), Laboratory of Experimental Medicine and Department of Endocrinology, Erasme \\ Hospital, Université Libre de Bruxelles, B-1070 Brussels, Belgium
}

\begin{abstract}
The aim of this study was to investigate, in normal young men, whether gamma-hydroxybutyrate (GHB), a reliable stimulant of slow-wave (SW) sleep in normal subjects, would simultaneously enhance sleep related growth hormone (GH) secretion. Eight healthy young men participated each in four experiments involving bedtime oral administration of placebo, 2.5, 3.0, and $3.5 \mathrm{~g}$ of GHB. Polygraphic sleep recordings were performed every night, and blood samples were obtained at 15-min intervals from 2000 to 0800 . GHB effects were mainly observed during the first $2 \mathrm{~h}$ after sleep onset. There was a doubling of GH secretion, resulting from an increase of the amplitude and the duration of the first GH pulse after sleep onset. This stimulation of GH secretion was significantly correlated to a simultaneous increase in the amount of sleep stage IV. Abrupt but transient elevations of prolactin and cortisol were also observed, but did not appear to be associated with the concomitant stimulation of SW sleep. Thyrotropin and melatonin profiles were not altered by GHB administration. These data suggest that pharmacological agents that reliably stimulate SW sleep, such as GHB, may represent a novel class of powerful GH secretagogues. (J. Clin. Invest. 1997. 100:745-753.) Key words: cortisol • prolactin • delta waves • aging • growth hormone-releasing hormone (GHRH)
\end{abstract}

\section{Introduction}

In normal young adults, the 24-h profile of growth hormone $(\mathrm{GH})^{1}$ secretion consists of low levels abruptly interrupted by large secretory pulses (1). The major secretory pulse usually occurs shortly after sleep onset, in temporal association with the

Address correspondence to Eve Van Cauter, Ph.D., Department of Medicine, MC 1027, University of Chicago, 5841 South Maryland Avenue, Chicago, IL 60637. Phone: 773-702-0169; FAX: 773-702-9194; E-mail: evcauter@medicine.bsd.uchicago.edu

Received for publication 24 February 1997 and accepted in revised form 24 April 1997.

1. Abbreviations used in this paper: GABA, gamma-aminobutyric acid; GH, growth hormone; GHB, gamma-hydroxybutyrate; GHRH, growth hormone-releasing hormone; IGF-I, insulin-like growth factor I; IGFBP-3, insulin-like growth factor-binding protein-3; REM, rapid-eye movement; $\mathrm{SW}$, slow-wave.

J. Clin. Invest.

(C) The American Society for Clinical Investigation, Inc. 0021-9738/97/08/0745/09 \$2.00

Volume 100, Number 3, August 1997, 745-753

http://www.jci.org first episode of slow-wave (SW) sleep $(2,3)$. A study with blood sampling at 30-s intervals has shown that maximal GH release occurs within minutes of the onset of SW sleep (4). Available evidence suggests that nocturnal GH secretion is controlled primarily by growth hormone-releasing hormone (GHRH) release (5). Because GH secretion is also under inhibitory control by somatostastin, variability of somatostatinergic tone may underlie dissociations between SW sleep and nocturnal GH release (6-9). While such dissociations are observed frequently during late sleep, a large pulse of GH secretion occurs during the first SW period $>90 \%$ of the time and there is a quantitative relationship between the duration of SW stages and the simultaneous amount of GH secreted (10). This latter observation raises the possibility that compounds that increase SW sleep may also be powerful GH secretagogues.

Commercially available hypnotics tend to inhibit, rather than increase, SW sleep and do not stimulate GH release (11-13). However, reliable stimulation of SW sleep in normal subjects has been obtained with oral administration of low doses of gamma-hydroxybutyrate (GHB), a simple four-carbon fatty acid that is used as an investigational drug for the treatment of narcolepsy (14-19). GHB is a metabolite of gamma-aminobutyric acid (GABA) that is normally present in the mammalian brain and is thought to be acting as a neurotransmitter (20-22). GHB readily crosses the blood-brain barrier but has a short duration of action, which limits its use for the treatment of insomnia.

The use of an orally active compound that could increase SW sleep and GH release simultaneously could be of potential benefit in older adults because aging is associated with marked decreases in both the duration of SW stages and the amount of GH secretion (23-26) but the temporal relationship between sleep onset and GH release is generally maintained (23). While the clinical implications of decreased SW sleep are unclear, multiple studies have indicated that the relative $\mathrm{GH}$ deficiency of the elderly is associated with increased fat tissue and abdominal obesity, reduced muscle mass and strength, and reduced exercise capacity (27-29). If compounds that stimulate SW sleep were able to also stimulate nocturnal GH secretion, they would represent a novel approach to increase endogenous GH secretion in older adults via the pharmacological enhancement of a physiological stimulus acting at a normal time of day.

Therefore, the aim of this study was to determine whether oral administration of relatively low doses of GHB at bedtime would enhance SW sleep and GH secretion simultaneously in normal young men.

\section{Methods}

Subjects

Eight healthy nonobese young men (age: $26 \pm 2 \mathrm{yr}$; body mass index (BMI): $23.5 \pm 0.7 \mathrm{~kg} / \mathrm{m}^{2}$; mean $\pm \mathrm{SEM}$ ) were included in the study after 
a careful clinical and biological evaluation. The subjects did not take any drugs and had no personal history of endocrine or sleep disorders. Shift workers and subjects having traveled across time zones within the previous 6 wk were excluded from the study. Positive criteria for selection included regular life habits and sleep schedules. The protocol was approved by the Institutional Review Board of the University of Chicago and all subjects gave written informed consent.

\section{Experimental protocol}

All investigations were performed in the Clinical Research Center (CRC) of the University of Chicago. Before the beginning of the study, the subjects were required to sleep two consecutive nights in the sleep laboratory of the Center to become habituated to the experimental environment. Throughout the entire study, the subjects agreed to maintain regular sleep-wake and meal schedules (bedtime: 2300-0700; breakfast: 0800; lunch: 1230; dinner: 1900).

Seven of the eight subjects participated in four studies involving bedtime oral administration of placebo, 2.5, 3.0, and $3.5 \mathrm{~g}$ of GHB. These dosages are used commonly in the treatment of narcolepsy (17, 18). One subject completed only the placebo and $2.5 \mathrm{~g}$ GHB studies. The studies were separated by $7 \pm 1 \mathrm{~d}$. The order of the studies was randomized and the subjects were blind to the experimental condition. For each study, the subjects were admitted at 1800 on two consecutive days and remained overnight in the CRC. No food was allowed throughout this period but water and noncaffeinated diet beverages were available. Naps were not allowed during waking hours. On both nights, placebo or GHB (Sigma Chemical Co., St. Louis, MO; available for FDA approved investigational purposes only) was administered orally at 2245 . The drug was administered under supervision as a single dose of $25 \mathrm{ml}$ of aqueous solution. The placebo preparation was similar in size, appearance and taste to the GHB preparation. On both nights, bedtimes were $2300-0700$ in total darkness and sleep was polygraphically recorded.

On one of the two nights, selected at random, a catheter was inserted in an antecubital vein within 15 min of the subject's admission. Blood samples (2.5 ml) for $\mathrm{GH}$, prolactin, cortisol, thyrotropin (TSH), and melatonin determinations were collected at 15-min intervals from 2000 to 0800 . An additional blood sample was also obtained at 0745 for the measurement of plasma insulin-like growth factor I (IGF-I) and insulin-like growth factor-binding protein-3 (IGFBP-3). The intravenous line was kept patent with a slow drip $(10 \mathrm{ml} / \mathrm{h})$ of heparinized saline ( $750 \mathrm{IU}$ heparin in $0.9 \mathrm{~g} \mathrm{NaCl} / \mathrm{dL}$ ). During bedtime hours, the indwelling catheter was connected to plastic tubing extending to an adjacent room, in order to collect blood samples without disturbing the subject. Blood samples were centrifuged at $4^{\circ} \mathrm{C}$. Plasma samples were kept frozen at $-20^{\circ} \mathrm{C}$ until assay.

\section{Hormonal assays}

GH levels were measured using a commercially available immunoradiometric assay (Sorin Biomedica, Milan, Italy) with a lower limit of sensitivity of $0.1 \mu \mathrm{g} /$ liter. The mean intraassay coefficient of variation was $7 \%$ in the range $0.2-1.0 \mu \mathrm{g} / \mathrm{liter}$ and $2.5 \%$ for concentrations above $1 \mu \mathrm{g} /$ liter. Prolactin levels were measured by a commercially available immunoradiometric assay (Medgenix, Fleurus, Belgium) with a limit of sensitivity of $0.35 \mu \mathrm{g} /$ liter. The mean intraassay coefficient of variation averaged $6.5 \%$. Cortisol levels were measured by a commercially available RIA (Coat-A-Count; Diagnostic Products Corporation, Los Angeles, CA) with a lower limit of sensitivity of 28 $\mathrm{nmol} /$ liter and an average intraassay coefficient of variation of $5 \%$. TSH levels were measured by a commercially available immunoradiometric assay (Medgenix) with a lower limit of sensitivity of 0.025 $\mathrm{mU} /$ liter and a mean intraassay coefficient of variation of $5 \%$. Plasma melatonin levels were measured after dichloromethane extraction with a double antibody RIA using an antibody against melatonin obtained from Stockgrand (Guilford, Surrey, UK). The lower limit of sensitivity of the assay is $2.5 \mathrm{pg} / \mathrm{ml}$. The coefficient of variation averaged $17.5 \%$ for concentrations below $10 \mathrm{pg} / \mathrm{ml}, 8.6 \%$ for concentrations in the range $10-30 \mathrm{pg} / \mathrm{ml}$, and $5.2 \%$ for concentrations $>30 \mathrm{pg} /$ ml. For all hormones measured in overnight studies, all samples obtained in the same individual study were analyzed in the same assay. IGF-I levels were determined by a commercially available RIA that involves an acid-ethanol extraction procedure (Nichols Institute Diagnostics, San Juan Capistrano, CA). The sensitivity of the assay is 15 $\mu \mathrm{g} / \mathrm{liter}$ and the mean intraassay coefficient of variation is $3 \%$. Plasma IGFBP-3 levels were measured by a commercially available immunoradiometric assay (Nichols Institute Diagnostics), with a lower limit of sensitivity of $0.6 \mathrm{mg} /$ liter and an intraassay coefficient of variation of $<4 \%$.

\section{Sleep recording and analysis}

Polygraphic sleep recordings were visually scored at 30-s intervals in stages wake, I, II, III, IV, and rapid-eye-movement (REM) using standardized criteria (30) by an experienced scorer who was blind to the study condition. The sleep period was defined as the time interval separating sleep onset from morning awakening. Sleep efficiency was calculated as the total recording time minus the total duration of awakenings, expressed in percent of the total recording time. SW stages were defined as the sum of stages III and IV. The first nonREM period was defined as the time interval between sleep onset and the first epoch of wake or REM, provided that the wake or the REM stage was maintained for at least $1 \mathrm{~min}$.

In addition, for 18 of the nights with blood sampling and for 19 of the nights without blood sampling, the EEG signal was sampled at $60 \mathrm{~Hz}$ by an analog/digital converter directly linked to a computer system and a computerized analysis of the EEG was performed using previously described procedures $(31,32)$. In each 20 -s epoch of recording, SW sleep was characterized by the spectral density in the standard delta frequency band $0.5-3 \mathrm{~Hz}$. In addition, typical delta activity, referred to as delta count, was examined in each 2.5 -s recording interval by fitting of a fourth order autoregressive all-pole model (31).

\section{Data analysis}

GH profiles. Significant pulses of GH secretion were identified using a modification of the computer algorithm ULTRA (33). The threshold for significance of a pulse was set at two times the intraassay coefficient of variation in the relevant range of concentration. For each significant pulse, the amount of GH secreted was estimated by deconvolution based on a one compartment model for GH clearance and variable individual half-lives, as described previously (10). The halflife was adjusted for each subject in the previously reported physiological range of 15-21 min (34) by an iterative process designed to minimize the number of negative secretory rates. On average, the half-disappearance time was $15.4 \pm 1.9 \mathrm{~min}$ (mean $\pm \mathrm{SD})$. A volume of distribution of $7 \%$ of body weight was used in these calculations. The standard deviation of the error associated with each estimated secretory rate was calculated according to the theory of error propagation and under the assumption of normally distributed errors on plasma levels. The duration of a secretory pulse was defined as the time interval separating the preceding and following troughs. The total amount of GH secreted over a given time interval was determined by summing the amounts secreted in each of the significant pulses occurring during that time interval. If a pulse overlapped two time intervals, the amount of GH secreted was divided proportionally.

Prolactin profiles. The secretion rate of prolactin was derived mathematically from plasma levels by deconvolution, using a onecompartment model for clearance kinetics with a fixed half-life of 25 min and a volume of distribution of $1587 \mathrm{ml} / \mathrm{m}^{2}$ of body surface area $(35,36)$. Significant pulses of prolactin secretion were identified using a modification of the computer algorithm ULTRA using the standard deviation of the error on each secretory rate to derive the threshold for pulse significance.

Cortisol profiles. Cortisol secretory rates were mathematically derived from the plasma cortisol concentrations using a two-compartment model for cortisol distribution and metabolism (37). The volume of distribution, short half-life, and fraction of decay associated with the short half-life were taken to be $4.1 \%$ of body weight, 5 min, 


\begin{tabular}{|c|c|c|c|c|c|}
\hline & Placebo & $2.5 \mathrm{~g} \mathrm{GHB}$ & $3.0 \mathrm{~g} \mathrm{GHB}$ & $3.5 \mathrm{~g} \mathrm{GHB}$ & ANOVA \\
\hline & $n=8$ & $n=8$ & $n=7$ & $n=7$ & \\
\hline Sleep latency (min) & $24 \pm 5$ & $13 \pm 3 *$ & $16 \pm 7$ & $14 \pm 2 *$ & $P<0.04$ \\
\hline Sleep period $(\min )$ & $447 \pm 6$ & $453 \pm 4$ & $441 \pm 20$ & $441 \pm 20$ & ns \\
\hline Sleep efficiency (\%) & $89 \pm 1$ & $83 \pm 4$ & $87 \pm 3$ & $80 \pm 4 *$ & $P<0.09$ \\
\hline Amount of Wake (min) & $21 \pm 5$ & $55 \pm 18$ & $43 \pm 12$ & $52 \pm 14$ & ns \\
\hline Amount of I + II (min) & $231 \pm 10$ & $224 \pm 10$ & $236 \pm 15$ & $223 \pm 18$ & ns \\
\hline Amount of III + IV (min) & $91 \pm 9$ & $88 \pm 8$ & $105 \pm 9$ & $99 \pm 18$ & ns \\
\hline Amount of IV (min) & $46 \pm 8$ & $45 \pm 10$ & $64 \pm 9$ & $59 \pm 19$ & ns \\
\hline Amount of REM (min) & $104 \pm 5$ & $84 \pm 16$ & $79 \pm 6^{*}$ & $64 \pm 7 * \neq$ & $P<0.005$ \\
\hline
\end{tabular}

${ }^{*} P<0.03$ versus placebo; ${ }^{\ddagger} P<0.02$ versus $2.5 \mathrm{~g} \mathrm{GHB}$ (pairwise contrasts tested by the Fisher procedure.)

and 0.80 , respectively, for all subjects (37). The long half-life was adjusted for each subject in the previously reported physiological range of 65-90 min by an iterative process designed to minimize the number of negative secretory rates (38). On average, the long half-life was $78 \pm 5$ min (mean $\pm S D)$. Significant pulses of cortisol secretion were identified using the same procedure as for prolactin.

Statistical tests. All comparisons between placebo, 2.5, 3.0, and $3.5 \mathrm{~g}$ of GHB were performed by ANOVA for repeated measures with pairwise contrasts tested by the Fisher procedure. Paired comparisons were performed by paired $t$ tests. All statistical calculations were performed using the Statview ${ }^{\mathrm{SE}+}$ software (Abacus Concepts, Inc., Berkeley, CA) for the Macintosh computer. Unless otherwise stated, all results are expressed as mean \pm SEM.

\section{Results}

Five of the subjects reported feeling inebriated after ingesting the highest GHB dosage. No other side effects were observed. Except for the $3.5 \mathrm{~g}$ dose, the subjects were unable to correctly identify whether they had received placebo or GHB. Consistent with previous reports $(14,39)$, paradoxical delta wave activity was observed before sleep onset in 8 of the 22 nights with GHB administration.

Sleep. For all sleep parameters, there were no significant differences between the night with and the night without blood sampling and, therefore, the data obtained in each subject for each study during the two successive nights were averaged before calculating group values.

Table I gives mean sleep parameters across the entire night for each study condition. The sleep latency was shortened after
GHB administration as compared to placebo. The sleep period was not affected by any of the doses of GHB. The amount of wake tended to increase after each dose of GHB as compared to placebo, but this effect was not sufficiently consistent to be statistically significant. Sleep efficiency was reduced as compared to placebo nights only for the highest GHB dosage. The total amount of stages I + II was not affected by any of the doses of GHB. In contrast, a significant reduction in the amount of REM sleep was observed with the two higher GHB doses. Over the entire night, there was no significant increase in amount of SW stages or amount of stage IV. However, examination of the temporal distribution of SW stages across the night (illustrated in the upper panel of Fig. 1) suggested that the effects of GHB on SW sleep were confined to the beginning of the sleep period, consistent with the short duration of action of the drug. A detailed analysis of the effects of GHB on SW sleep during the beginning of the sleep period was thus performed and is summarized in Table II.

On average, the amounts of stages IV and III + IV during the initial $2 \mathrm{~h}$ of sleep were increased by GHB administration, but this increase was significant only for stage IV after the medium dose of the drug. Both the mean delta count and the mean spectral density in the delta range (percentage delta power) were increased significantly during the first $2 \mathrm{~h}$ of sleep after the highest GHB dose. At each dosage, GHB administration resulted in a prolongation of the first non-REM period. During this first non-REM period, the total amount of stages IV and III + IV were increased after the two higher GHB doses as compared to placebo.
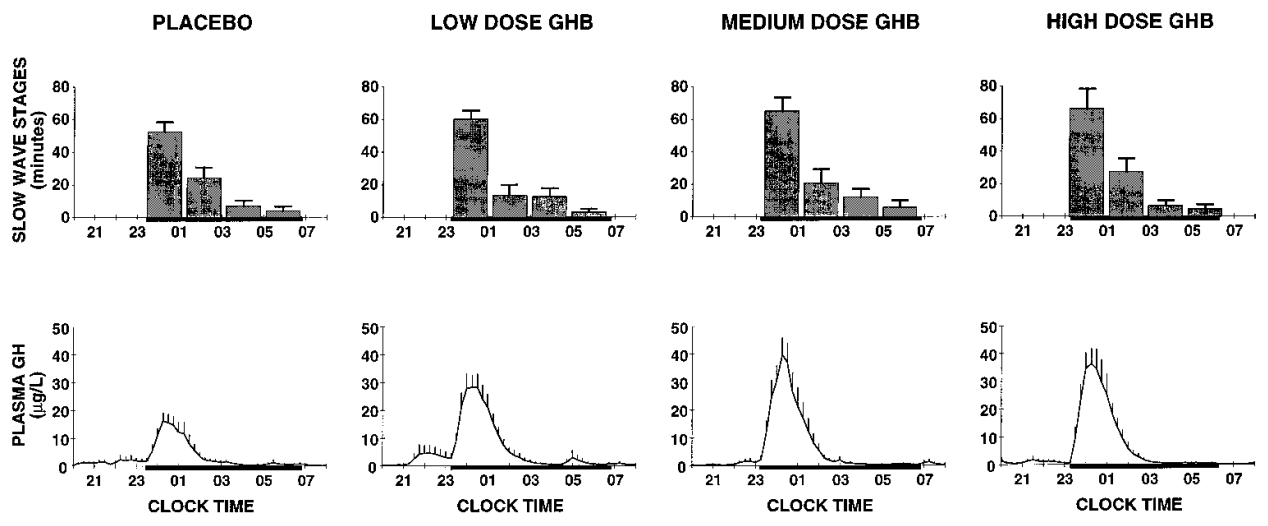

Figure 1. Mean (+ SEM) profiles of SW sleep and plasma GH in the four treatment conditions. The drug was given at 2245. The amounts of SW sleep (upper panel) are shown for successive 2-h periods from 2300 to 0700 . Black bars denote the sleep periods. 
Table II. Effects of GHB on SW Sleep during the Beginning of the Sleep Period (Mean \pm SEM)

\begin{tabular}{|c|c|c|c|c|c|}
\hline & Placebo & $2.5 \mathrm{~g} \mathrm{GHB}$ & $3.0 \mathrm{~g} \mathrm{GHB}$ & $3.5 \mathrm{~g} \mathrm{GHB}$ & ANOVA \\
\hline & $n=8$ & $n=8$ & $n=7$ & $n=7$ & \\
\hline \multicolumn{6}{|l|}{ First $2 \mathrm{~h}$} \\
\hline Amount of III + IV (min) & $49 \pm 5$ & $54 \pm 6$ & $67 \pm 5$ & $63 \pm 11$ & ns \\
\hline Amount of IV (min) & $26 \pm 5$ & $32 \pm 7$ & $51 \pm 6^{*}$ & $42 \pm 12$ & ns \\
\hline Mean delta count per 20-s epoch & $2.1 \pm 0.6$ & - & - & $3.4 \pm 0.8$ & $P<0.05$ \\
\hline Mean percentage delta power in 20 -s epoch & $59.5 \pm 4.2$ & - & - & $67.8 \pm 4.4$ & $P<0.05$ \\
\hline \multicolumn{6}{|l|}{ First non-REM period } \\
\hline Duration (min) & $69 \pm 10$ & $89 \pm 8^{*}$ & $132 \pm 12 * \neq$ & $117 \pm 15^{*}$ & $P<0.001$ \\
\hline Amount of SW stages (min) & $34 \pm 5$ & $48 \pm 5$ & $57 \pm 8^{\ddagger}$ & $63 \pm 14^{\ddagger}$ & $P<0.04$ \\
\hline Amount of IV (min) & $21 \pm 5$ & $29 \pm 8$ & $49 \pm 9 *$ & $47 \pm 14^{*}$ & ns \\
\hline
\end{tabular}

${ }^{*} P<0.05$ versus placebo; ${ }^{*} P<0.03$ versus placebo; ${ }^{\S} P<0.05$ versus $2.5 \mathrm{~g} \mathrm{GHB} ;-$ insufficient number of computerized recordings.

Growth hormone. Fig. 1 shows mean profiles of plasma $\mathrm{GH}$ levels, calculated across subjects for each treatment condition, together with the amount of SW stages over successive 2-h periods after sleep onset. Group parameters quantifying $\mathrm{GH}$ secretion in each treatment condition are given in Fig. 2. The number of nocturnal GH pulses was similar in all four study conditions, averaging $5.0 \pm 0.4$ over the period $2300-0700$. The total amount of GH secreted over the same time period was consistently higher after each dose of GHB than after placebo administration $(P<0.004)$. Values were similar after the two higher doses (3.0 and $3.5 \mathrm{~g}$ ), and were approximately twofold higher than after placebo. This elevation of nocturnal GH secretion was mainly due to a robust increase in the height and duration of the first GH pulse after sleep onset (Figs. 1 and 2). Not surprisingly, plasma IGF-I and IGFBP-3 levels, measured $\sim 9 \mathrm{~h}$ after drug ingestion (i.e., $7-8 \mathrm{~h}$ after the major secretory pulse) were not altered by GHB administration (Fig. 2).

The parallelism between the enhancement of SW sleep and the increase in GH levels induced by GHB during the first $2 \mathrm{~h}$ of sleep is apparent in Fig. 1. Although the increase in GH secretion was markedly more pronounced than the increase in SW sleep, analysis of hormonal and sleep alterations during the first non-REM period indicated the existence of a consistent association between GHB-induced stimulation of stage IV and of GH secretion ( $r=0.73, P=0.0001$, Fig. 3$)$. This correlation persisted when the first $2 \mathrm{~h}$ of sleep, rather than the first non-REM period, were considered $(r=0.48, P<0.03)$, indicating that GHB-induced GH stimulation did not exclusively reflect the prolongation of the first non-REM period.

Fig. 4 illustrates the mean onsets of the major GH secretory pulse under the four study conditions relative to the time of drug administration (i.e., 22:45; left panel) and relative to the time of sleep onset (defined as the timing of the first page of stage II; right panel). GHB treatment tended to be associated with an advance of the onset of $\mathrm{GH}$ secretion relative to time of drug ingestion $(P<0.06)$. Effects of GHB on the delay between sleep onset and onset of GH secretion were nonsignificant, except for the $3 \mathrm{~g}$ GHB condition $(P=0.05)$ because of the fact that, after this dosage, one of the subjects initiated $\mathrm{GH}$ secretion nearly one hour before sleep onset. This was the only instance of GH secretion before sleep onset and it was not associated with concomitant paradoxical delta wave activity.

Prolactin. Mean profiles of plasma prolactin levels for each treatment condition are shown in Fig. 5. Within 30 min, each dose of GHB induced a rapid and large increase of prolactin levels, contrasting with the progressive elevation throughout the sleep period observed under placebo. On average, the maximal nocturnal increment of plasma prolactin above daytime level was more than twofold higher after GHB than after placebo (Fig. 5). During the time interval 2300-0300, prolactin secretion was markedly enhanced after GHB administration (placebo: 170 $\pm 16 \mu \mathrm{g}$; $2.5 \mathrm{~g}$ GHB: $240 \pm 30 \mu \mathrm{g} ; 3 \mathrm{~g}$ GHB: $295 \pm 25 \mu \mathrm{g}$; $3.5 \mathrm{~g}$ GHB: $315 \pm 28 \mu \mathrm{g} ; P<0.0001)$. In contrast, during the later part of the night, GHB treatment was associated with lower levels of prolactin secretion than placebo (placebo: $218 \pm 22 \mu \mathrm{g} ; 2.5 \mathrm{~g}$ GHB: $184 \pm 28 \mu \mathrm{g} ; 3 \mathrm{~g}$ GHB: $194 \pm 28 \mu \mathrm{g}$; $3.5 \mathrm{~g} \mathrm{GHB}: 178 \pm 25 \mu \mathrm{g} ; P<0.02)$.

In contrast with $\mathrm{GH}$, there was no relationship between the enhancement of stage IV sleep and the increase in prolactin secretion induced by GHB during the first non-REM period ( $r=$ 0.09 , ns), indicating that GHB stimulation of prolactin secretion was not associated with the concomitant stimulation of SW sleep. Moreover, and also in contrast with GH, the onset of prolactin secretion, whether expressed relative to the timing of drug administration or relative to the timing of sleep onset, was significantly advanced after all doses of GHB as compared to placebo $(P<0.02$ at least $)$, and occurred before sleep onset in the majority of studies (Fig. 4).

Cortisol. Mean profiles of plasma cortisol levels for each treatment condition are shown in Fig. 5. Under placebo, mean cortisol profiles conformed with the normal pattern of cortisol secretion, with low levels of secretion in the late evening and in the beginning of the sleep period, followed by an abrupt early morning elevation. Administration of 3 and $3.5 \mathrm{~g}$ GHB was associated in all individuals with a well-defined elevation of cortisol secretion over the $2300-0300$ period (placebo: $1.58 \pm 0.31$ mmol; $3 \mathrm{~g} \mathrm{GHB:} 4.19 \pm 0.63 \mathrm{mmol}, P<0.01$ versus placebo; $3.5 \mathrm{~g}$ GHB: $4.63 \pm 0.76 \mathrm{mmol}, P<0.01$ versus placebo). With the $2.5 \mathrm{~g}$ dose, a similar increase in cortisol secretion occurred in five of eight subjects over the same time period. Over the 0300-0800 period, cortisol secretion was not altered with the two lower doses, and was decreased significantly with the higher dose, as compared with placebo.

Pulse analysis of the profiles of cortisol secretory rates indicated that, during the 2-h period after drug intake, GHB administration was associated with a higher pulse frequency than 

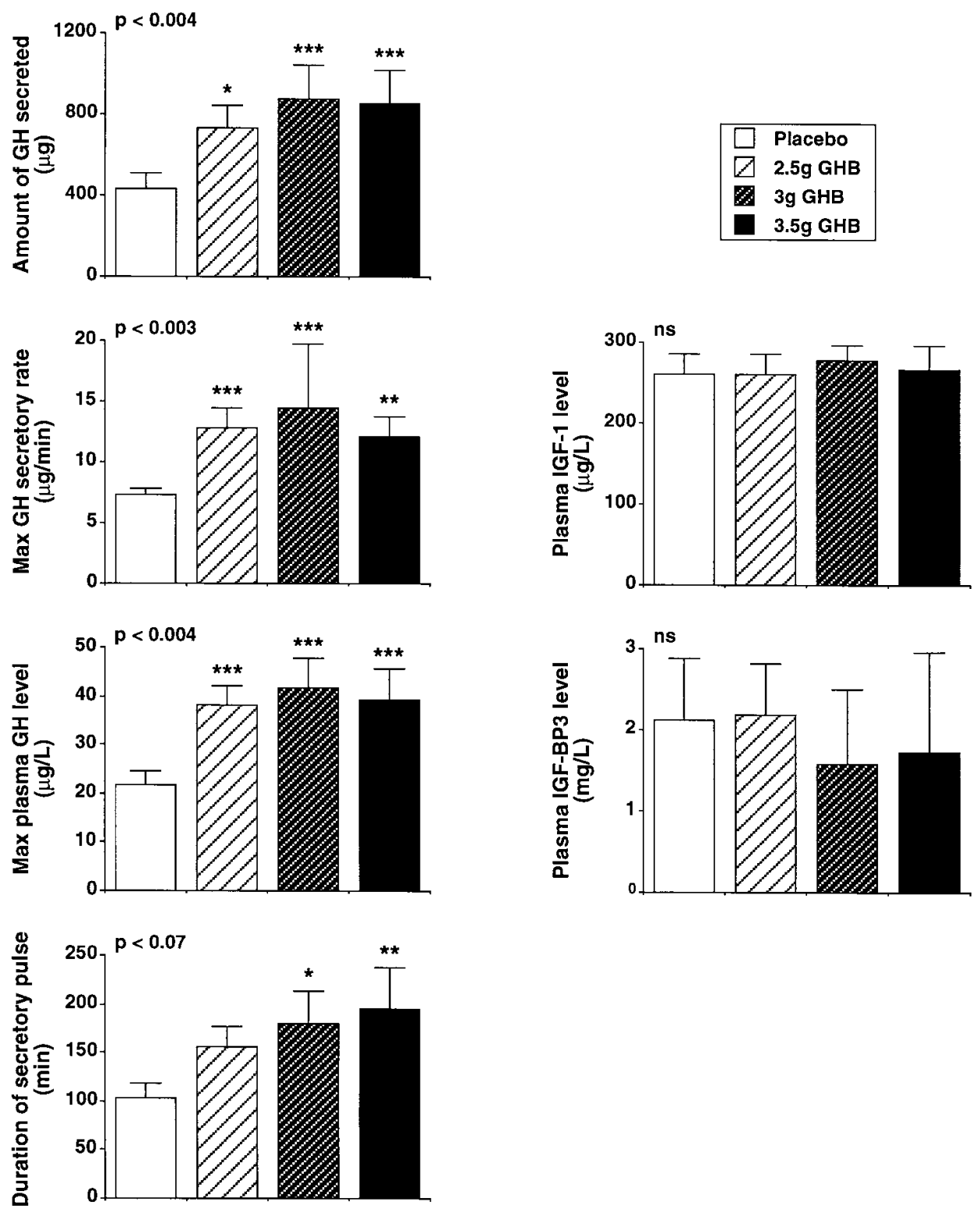

Figure 2. Mean (+ SEM) values in each treatment condition of parameters quantifying $\mathrm{GH}$ secretion over the period $2300-0700$, and of plasma levels of IGF-I and IGFBP-3 at 0745 . $P$ values shown in each panel denote the results of ANOVA calculations. Asterisks denote the results of pairwise contrasts tested by the Fisher procedure, as compared to placebo: ${ }^{*} P<$ $0.05 ; * P<0.02 ; * * * P<0.005$.

placebo $(P<0.04)$. Analysis of the temporal distribution of pulsatile secretion exceeding $0.5 \mathrm{mmol}$ (illustrated in Fig. 4) indicated that in $23 \%$ of the studies, GHB ingestion was followed by two consecutive pulses of cortisol secretion, one occurring within $45 \mathrm{~min}$ and the other occurring 60-120 min later. In $27 \%$ of the studies, only the early pulse was observed, whereas in $41 \%$ of the studies, only the later pulse was present. Only in 2 of the 22 profiles (i.e., $9 \%$ ), no cortisol pulse was detected within two hours post-GHB and both cases corresponded to the lowest dosage. Early and late pulses accounted for 36 and $64 \%$, respectively, of the increment in cortisol secretion observed after GHB administration. The majority of early cortisol pulses post-GHB were initiated before sleep onset. There were no significant effects of GHB on cortisol pulse frequency in the later part of the night.

TSH and melatonin. Mean profiles of plasma TSH and melatonin levels are shown in the two lower panels of Fig. 5. For both hormones, normal profiles were observed after pla- cebo administration and were not altered by any of the doses of GHB.

\section{Discussion}

The present study demonstrates that bedtime administration of GHB, even at the lowest dose, results in a twofold increase in the amount of $\mathrm{GH}$ secreted during sleep. This effect of $\mathrm{GHB}$ on nocturnal $\mathrm{GH}$ secretion resulted from an increase in the amplitude and the duration of the normal secretory pulse associated with sleep onset, rather than from the induction of additional pulses. The increase in GH secretion was only initiated after the first epoch of stage II sleep had been recorded and was correlated quantitatively with an increase in amount of stage IV during early sleep. Consistent evidence has been reported in a recent study where a $24 \%$ increase in delta wave activity was paralleled by a $29 \%$ increase in $\mathrm{GH}$ secretory rate after sleep onset (40). These findings indicate the existence of 


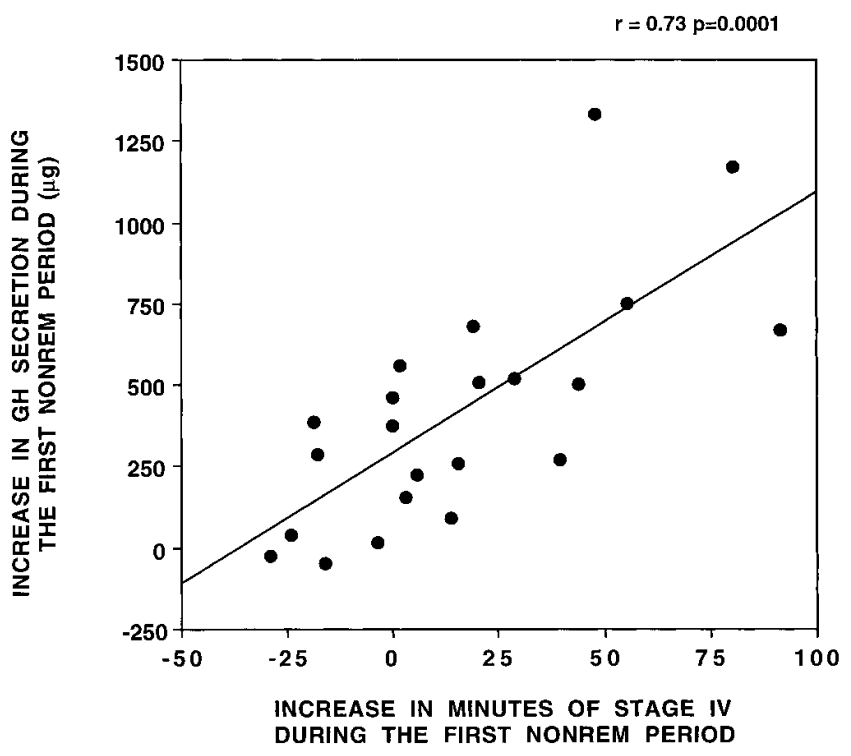

Figure 3. Correlation between increases in the amount of stage IV during the first nonREM period after GHB administration, and corresponding increases in $\mathrm{GH}$ secretion during the same period.

a robust relationship between the deeper stages of sleep and $\mathrm{GH}$ release in the human, supporting the concept that common pathways are involved in the control of SW sleep and nocturnal GH release (41). The remarkable correlation between increased SW sleep and augmented GH release indicates that pharmacological agents which reliably stimulate SW sleep may represent a novel class of powerful GH secretagogues.

Effects of GHB on sleep parameters were generally consistent with previous descriptions $(14-16,39)$. High voltage delta waves were observed occasionally before sleep onset, as reported previously (39), but were not associated with concomitant GH secretion, suggesting that GHB will only act as a $\mathrm{GH}$ secretagogue if sleep is induced. REM sleep appeared after a normal latency but the total amount of REM was decreased. This decrease in total REM sleep was probably due to the fact that the amount of wake tended to increase although this effect failed to reach statistical significance. Indeed, many subjects experienced an awakening after $2-3 \mathrm{~h}$ of sleep, when the effects of the drug started waning.

Treatment with GHB was otherwise very well-tolerated and in the majority of studies, the subjects were unable to determine whether they had received GHB or placebo. The only side effect was a feeling of inebriation that occurred only with the highest dosage. These observations are consistent with previous observations from clinical research studies published over the past 20 years that have included a total of nearly 200 subjects (14-19, 42-45) as well as with the experience accumulated in an open clinical trial which has enrolled over 120 narcoleptic subjects treated nightly with 2-3 g doses of GHB for periods of up to 13 years. In this population, the only side effects have been episodes of enuresis and sleep walking and were observed in $<1 \%$ of the treated nights (M.B. Scharf, unpublished observations). In contrast to the low toxicity of GHB observed in controlled conditions, a number of mild to very severe adverse reactions (ranging from drowsiness and nausea to seizures and coma) after uncontrolled uses of variable doses of GHB, often in association with ethanol and/or other drugs, have been reported over the past few years (46-50).

The mechanisms underlying the stimulatory effects of bedtime administration of GHB on $\mathrm{GH}$ release remain to be defined. The temporal and quantitative associations between increased SW sleep and increased GH release observed in this study strongly suggest that the effects of GHB are exerted at the level of the central nervous system and are mediated, at

TIMING OF ONSET OF HORMONAL SECRETION

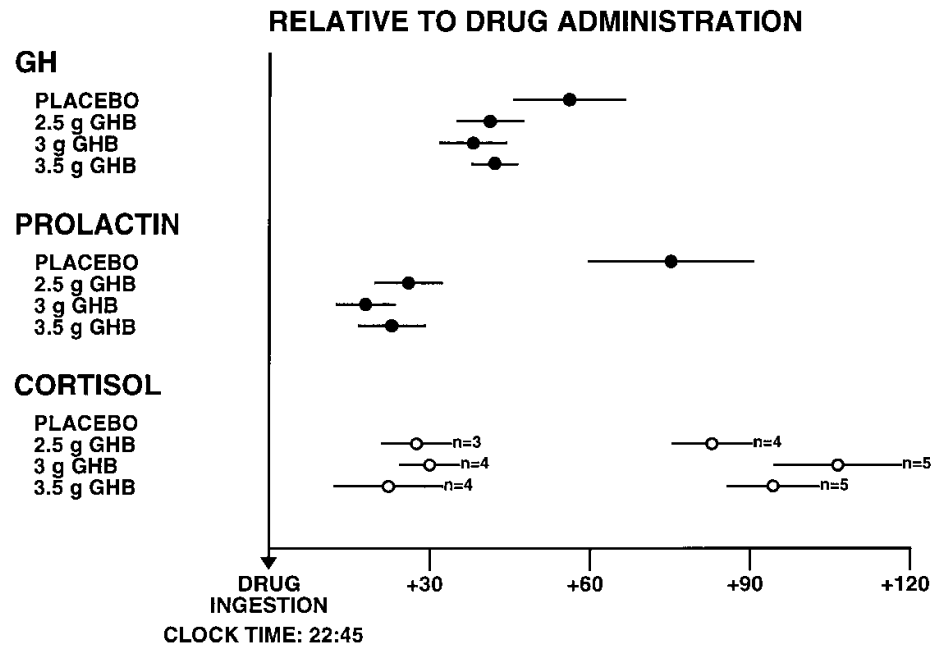

MINUTES

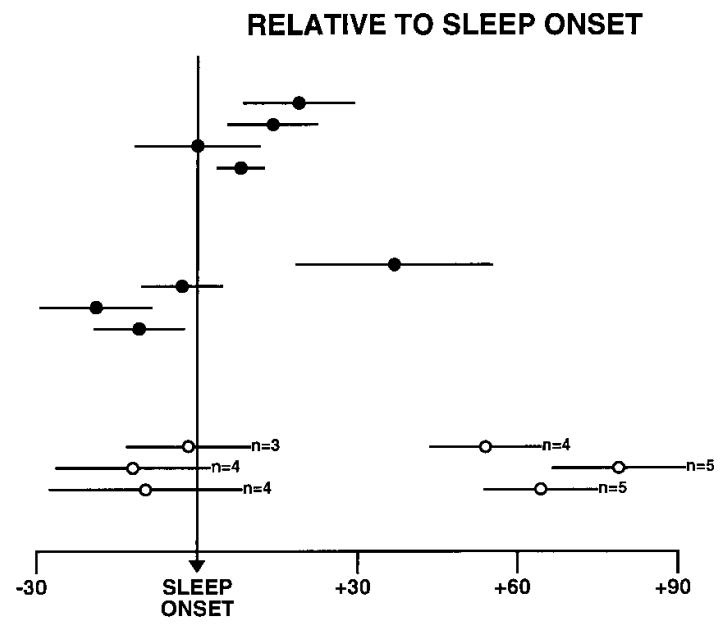

MINUTES

Figure 4. Mean ( \pm SEM) timing of the onset of GH, prolactin, and cortisol secretion after placebo or GHB administration (left) or after sleep onset (right). Under placebo conditions, only one subject (not illustrated) exhibited a cortisol pulse during the 120-min period after drug ingestion. Note that prolactin and cortisol secretion were generally initiated before GH secretion. Only in 1 of 22 studies with GHB administration was GH secretion initiated before sleep onset. 

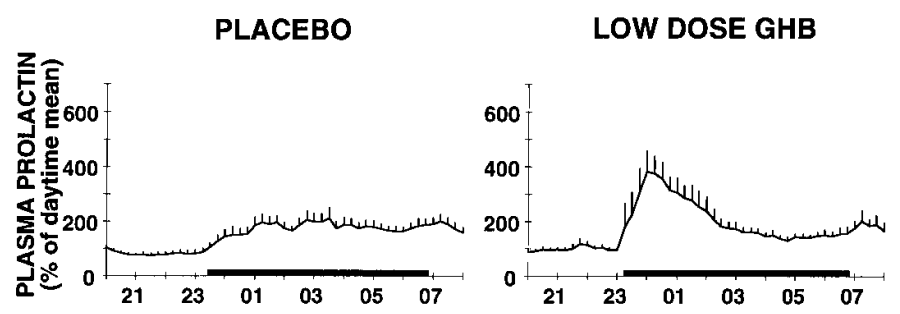

MEDIUM DOSE GHB

HIGH DOSE GHB
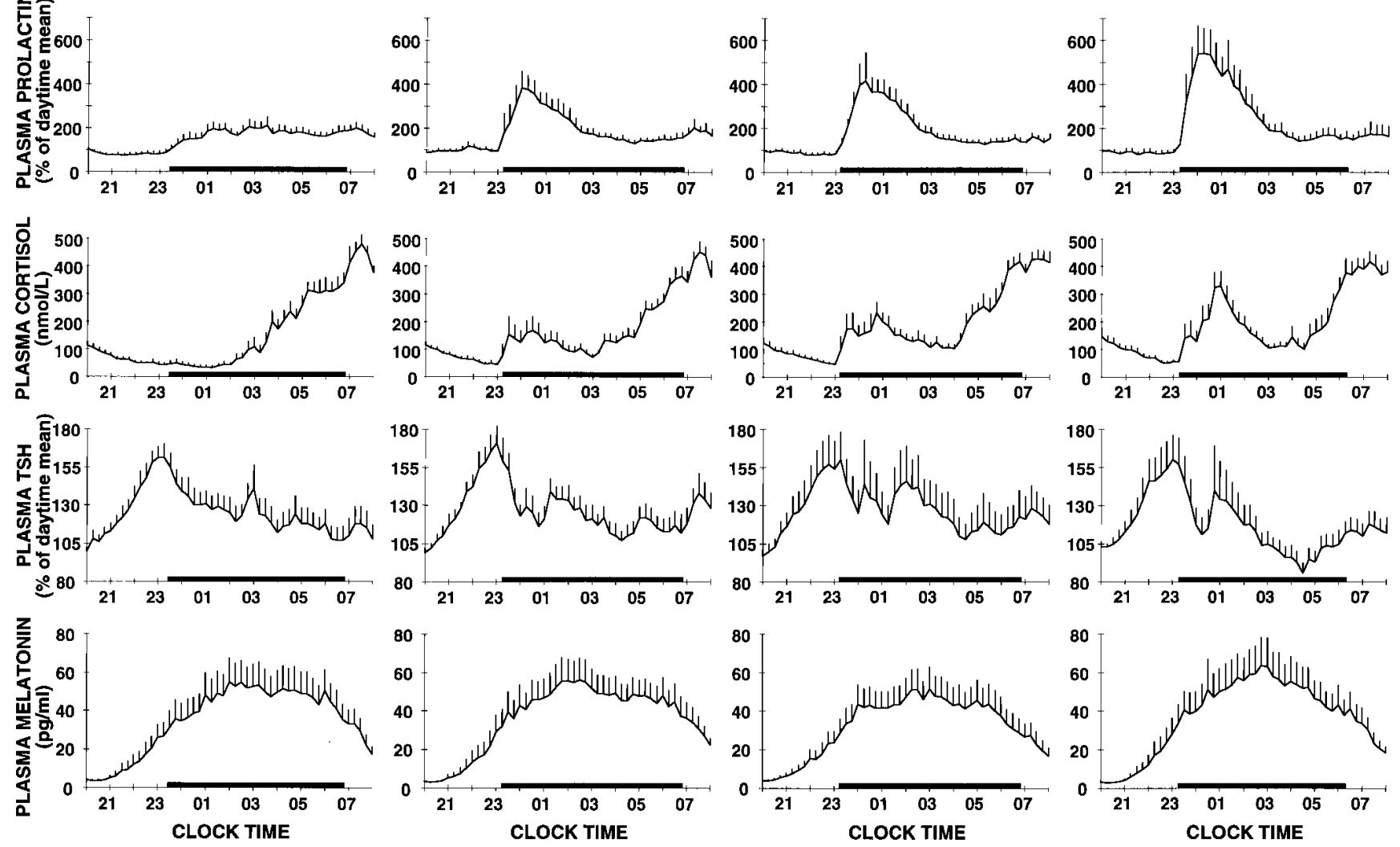

Figure 5. Mean (+ SEM) profiles of plasma prolactin, plasma cortisol, plasma TSH, and plasma melatonin in the four treatment conditions. To eliminate interindividual variability, prolactin, and TSH concentrations were expressed for each individual profile as a percentage of the mean value during the 2000-2300 time interval, or during the 2000-2100 time interval, respectively. The drug was given at 2245 . Black bars denote the sleep periods.

least partially, by the effects of GHB on sleep. Indeed, with only one exception, increases in GH secretory rate did not occur before sleep onset unlike elevations in prolactin secretory rate which generally preceded sleep onset. Two previous studies have examined effects of GHB on GH and prolactin secretion during wake but neither included EEG recordings (42, $44)$. In one early study (42), a dose of $2.5 \mathrm{~g}$ GHB was administered intravenously in the morning to healthy male volunteers and $\mathrm{GH}$ and prolactin levels were sampled at $15 \mathrm{~min}$ for $2 \mathrm{~h}$ post-GHB. Based on visual inspection, five of the six subjects fell asleep. Interestingly, prolactin levels had increased by nearly threefold $15 \mathrm{~min}$ after injection, but $\mathrm{GH}$ concentrations did not rise until $30 \mathrm{~min}$ after injection, suggesting that $\mathrm{GH}$ did not increase until sleep was initiated. A more recent study with oral administration of a very low dose $(1.5 \mathrm{~g})$ of GHB in the morning (44) is difficult to interpret because baseline plasma $\mathrm{GH}$ levels were markedly elevated. Studies involving oral administration of GHB and polygraphic recordings in subjects maintained awake will be necessary to determine whether GHB is capable of increasing GH secretion in the absence of sleep. Unpublished in vitro studies indicate that GHB does not bind to the recently identified GHRP/MK-0677 receptor (R.E. Smith, personal communication). Ongoing experiments in another laboratory are examining whether GHB binds to the pituitary GHRH receptor.
The neural pathways subserving the relationship between SW sleep and GH release are poorly understood. A recent study during overnight infusion of a GHRH antagonist has shown that the primary control of nocturnal GH secretion is through GHRH (5). Although sleep stages were not recorded, this study strongly suggests that GH secretion during SW sleep is mediated by GHRH release and therefore that the effects of GHB on GH secretion during sleep may involve an increased release of GHRH. Multiple actions of GHB on the GABAergic, cholinergic, serotoninergic, and dopaminergic systems have been described (20-22) and further studies will be needed to delineate the pathways that subserve the interactions between GHB and the central control of GH release.

At each dosage, GHB ingestion was rapidly followed by significant elevations of prolactin and cortisol secretion although plasma levels of both hormones remained within the physiologic range. The increase in prolactin secretion preceded the increase in GH secretion and was generally initiated before sleep onset. This rapid prolactin-enhancing effect of GHB is consistent with data from animal studies showing that, when administered systemically, the action of GHB seems to involve an initial decrease in dopamine release (20-22). Despite the fact that, under normal conditions, pulsatile prolactin secretion occurs preferentially during SW sleep (51), the enhancement of SW sleep is unlikely to have played a major role 
in the prolactin response since, in contrast to $\mathrm{GH}$, there was no correlation between the increase in prolactin secretion and the stimulation of SW sleep.

The increase in cortisol secretion associated with GHB treatment was less consistent and appeared to involve two separate phases of increased hypothalamo-pituitary-adrenal activity, one starting within $30 \mathrm{~min}$ after drug ingestion, i.e., frequently before sleep onset, and the other starting $\sim 1 \mathrm{~h}$ later, i.e., when drug concentrations and $\mathrm{GH}$ levels were decreasing rapidly. Since studies in rodents have shown that, in addition to its antidopaminergic properties, GHB may also interfere with cholinergic, serotoninergic, and GABAergic transmission (20-22), the acute release of cortisol post-GHB may have multiple hypothetical causes. The delayed effect of GHB on cortisol secretion, which represents roughly $65 \%$ of the total response, could be related to the short duration of action of the compound and could perhaps be eliminated with a longer acting preparation.

In normal older adults, both the total amount of SW stages and the daily amount of GH secretion are drastically decreased but the association between sleep onset and GH release is generally maintained (23). There is good evidence indicating that many catabolic changes seen in normal aging including decreased lean body mass, increased adiposity and perhaps osteoporosis may be in part caused by decreased GH secretion (27-29). Multiple studies (28, 52-55) have explored the potential beneficial effects of $\mathrm{GH}$ replacement with daily subcutaneous injections of recombinant human $\mathrm{GH}$, which result in an extended period of high plasma GH levels, in sharp contrast with the normal pulsatile pattern. It has been speculated that the side effects of GH therapy may be related to the nonphysiological concentration profile achieved in the peripheral circulation (56-58). The restoration of a youthful profile of endogenous $\mathrm{GH}$ secretion via the enhancement of SW sleep may therefore offer a better alternative than parenteral GH. Ongoing studies in our laboratory are examining the effects of prolonged (4 wk) daily administration of GHB in older adults to determine whether enhancement of SW sleep and concomitant GH secretion may be obtained in this population, and provide novel therapeutical strategies for the simultaneous treatment of relative growth hormone deficiency and sleep disturbances in the elderly.

\section{Acknowledgments}

The authors are indebted to the volunteers who participated in the study and to the nursing staff of the University of Chicago Clinical Research Center for expert assistance.

This work was supported in part by grants DK-41814 from the National Institute of Diabetes and Digestive and Kidney Diseases (National Institutes of Health), AG-11412 from the National Institute on Aging (NIH), and by the Mind-Body Network of the MacArthur Foundation (Chicago, IL). The University of Chicago Clinical Research Center is supported by NIH grant RR-00055.

\section{References}

1. Van Cauter, E., and L. Plat. 1996. Physiology of growth hormone during sleep. J. Pediatr. 128:S32-S37.

2. Takahashi, Y., D.M. Kipnis, and W.H. Daughaday. 1968. Growth hormone secretion during sleep. J. Clin. Invest. 47:2079-2090.

3. Sassin, J.F., D.C. Parker, J.W. Mace, R.W. Gotlin, L.C. Johnson, and L.G. Rossman. 1969. Human growth hormone release: relation to slow-wave sleep and sleep-waking cycles. Science (Wash. DC). 165:513-515.
4. Holl, R.W., M.L. Hartmann, J.D. Veldhuis, W.M. Taylor, and M.O. Thorner. 1991. Thirty-second sampling of plasma growth hormone in man: correlation with sleep stages. J. Clin. Endocrinol. Metab. 72:854-861.

5. Ocampo-Lim, B., W. Guo, R. DeMott Friberg, A.L. Barkan, and C.A. Jaffe. 1996. Nocturnal growth hormone (GH) secretion is eliminated by infusion of GH-releasing Hormone antagonist. J. Clin. Endocrinol. Metab. 81:43964399.

6. Jarrett, D.B., J.B. Greenhouse, J.M. Miewald, I.B. Fedorka, and D.J. Kupfer. 1990. A reexamination of the relationship between growth hormone secretion and slow wave sleep using delta wave analysis. Biol. Psychiatry. 27:497-509.

7. Born, J., S. Muth, and H.L. Fehm. 1988. The significance of sleep onset and slow wave sleep for nocturnal release of growth hormone $(\mathrm{GH})$ and cortisol. Psychoneuroendocrinology. 13:233-243.

8. McCracken, J.T., R.E. Poland, R.T. Rubin, and L. Tondo. 1991. Dosedependent effects of scopolamine on nocturnal growth hormones secretion in normal adult men: relation to $\delta$-sleep changes. J. Clin. Endocrinol. Metab. 72: 90-95.

9. Peters, J.R., P.J. Evans, M.D. Page, R. Hall, J.T. Gibbs, C. Dieguez, and M.F. Scanlon. 1986. Cholinergic muscarinic receptor blockade with pirenzepine abolishes slow-wave sleep-related growth hormone release in normal adult males. Clin. Endocrinol. 26:213-217.

10. Van Cauter, E., M. Kerkhofs, A. Caufriez, A. Van Onderbergen, M.O. Thorner, and G. Copinschi. 1992. A quantitative estimation of GH secretion in normal man: reproducibility and relation to sleep and time of day. J. Clin. Endocrinol. Metab. 74:1441-1450.

11. Scharf, M.B. 1992. Pharmacology of classic and novel hypnotic drugs. In Target Receptors for Anxiolytics and Hypnotics: From Molecular Pharmacology to Therapeutics. S.Z. Langer, J. Mendlewicz, and C. Racagni, editors. Karger, Basel. 109-116.

12. Copinschi, G., A. Van Onderbergen, M. L'Hermite-Balériaux, M. Szyper, A. Caufriez, D. Bosson, M. L'Hermite, C. Robyn, F.W. Turek, and E. Van Cauter. 1990. Effects of the short-acting benzodiazepine triazolam, taken at bedtime, on circadian and sleep-related hormonal profiles in normal men. Sleep (Rochester). 13:232-244.

13. Copinschi, G., E. Akseki, R. Moreno-Reyes, R. Leproult, M. L'Hermite-Balériaux, A. Caufriez, F. Vertongen, and E. Van Cauter. 1995. Effects of bedtime administration of zolpidem on circadian and sleep-related hormonal profiles in normal women. Sleep (Rochester). 18:417-424.

14. Mamelak, M., J.M. Escriu, and O. Stokan. 1977. The effects of gammahydroxybutyrate on sleep. Biol. Psychiatry. 12:273-288.

15. Lapierre, O., J. Montplaisir, M. Lamarre, and M.A. Bedard. 1990. The effects of gamma-hydroxybutyrate on nocturnal and diurnal sleep of normal subjects: further consideration on REM-sleep triggering mechanisms. Sleep (Rochester). 13:24-30.

16. Series, F., I. Series, and Y. Cormier. 1992. Effects of enhancing slowwave sleep by gamma-hydroxybutyrate on obstructive sleep apnea. Am. Rev. Respir. Dis. 145:1378-1383.

17. Mamelak, M., M.B. Scharf, and M. Woods. 1986. Treatment of narcolepsy with gamma-hydroxybutyrate. A review of clinical and sleep laboratory findings. Sleep (Rochester). 9:285-289.

18. Scharf, M.B., D. Brown, M. Woods, L. Brown, and J. Hirschowitz. 1985. The effects and effectiveness of gamma-hydroxybutyrate in patients with narcolepsy. J. Clin. Psychiatry. 46:222-225.

19. Lammers, G.J., J. Arends, A.C. Declerck, M.D. Ferrari, G. Schouwink, and J. Troost. 1993. Gammahydroxybutyrate and narcolepsy: a double-blind placebo-controlled study. Sleep (Rochester). 16:216-220.

20. Mamelak, M. 1989. Gamma-hydroxybutyrate: an endogenous regulator of energy metabolism. Neurosci. Biobehav. Rev. 13:187-198.

21. Tunnicliff, G. 1992. Significance of gamma-hydroxybutyrate in the brain. Gen Pharmacol. 23:1027-1034.

22. Cash, C.D. 1994. Gammahydroxybutyrate: an overview of the pros and cons for it being a neurotransmitter and/or a useful therapeutic agent. Neurosci. Biobehav. Rev. 18:291-304.

23. van Coevorden, A., J. Mockel, E. Laurent, M. Kerkhofs, M. L'HermiteBalériaux, C. Decoster, P. Nève, and E. Van Cauter. 1991. Neuroendocrine rhythms and sleep in aging. Am. J. Physiol. 260:E651-E661.

24. Veldhuis, J., A. Liem, S. South, A. Weltman, J. Weltman, D. Clemmons, R. Abbott, T. Mulligan, M. Johnson, S. Pincus, et al. 1995. Differential impact of age, sex steroid hormones, and obesity on basal versus pulsatile growth hormone secretion in men as assessed in an ultrasensitive chemiluminescence assay. J. Clin. Endocrinol. Metab. 80:3209-3222.

25. Vermeulen, A. 1987. Nyctohemeral growth hormone profiles in young and aged men: correlation with somatomedin-C levels. J. Clin. Endocrinol. Metab. 64:884-888.

26. Ho, K.Y., W.S. Evans, R.M. Blizzard, J.D. Veldhuis, G.R. Merriam, E. Samojlik, R. Furlanetto, A.D. Rogol, D.L. Kaiser, and M.O. Thorner. 1987. Effects of sex and age on the 24-hour profile of growth hormone secretion in man: importance of endogenous estradiol concentrations. J. Clin. Endocrinol. Metab. 64:51-58.

27. Cuneo, R., F. Salomon, G. McGauley, and P. Sonksen. 1992. The growth hormone deficiency syndrome in adults. Clin. Endocrinol. 37:387-397.

28. Corpas, E., S.M. Harman, and M.R. Blackman. 1993. Human growth 
hormone and human aging. Endocr. Rev. 14:20-39.

29. Rosen, T., T. Hansson, H. Granhed, J. Szucs, and B. Bengtsson. 1993. Reduced bone mineral content in adult patients with growth hormone deficiency. Acta. Endocrinol. 129:201-206.

30. Rechtschaffen, A., and A. Kales. 1968. A manual of standardized terminology, techniques and scoring system for sleep stages of human subjects. Government Printing Office, Washington DC.

31. Stanus, E., B. Lacroix, M. Kerkhofs, and J. Mendlewicz. 1987. Automated sleep scoring: a comparative reliability study of two algorithms. Electroencephalogr. Clin. Neurophysiol. 66:448-456.

32. Stanus, E.L., B.P. Lacroix, M. Kerkhofs, and J. Mendlewicz. 1988. Computer-aided sleep staging in clinical environment. Neuropsychobiology. 20:178-186.

33. Van Cauter, E. 1988. Estimating false-positive and false-negative errors in analyses of hormonal pulsatility. Am. J. Physiol. 254:E786-E794.

34. Faria, A.C.S., J.D. Veldhuis, M.O. Thorner, and M.L. Vance. 1989. Half-time of endogenous growth hormone $(\mathrm{GH})$ disappearance in normal man after stimulation of GH secretion by GH-releasing hormone and suppression with somatostatin. J. Clin. Endocrinol. Metab. 68:535-541.

35. Veldhuis, J.D., A. Iranmanesh, M.L. Johnson, and G. Lizarralde. 1990. Twenty-four-hour rhythms in plasma concentrations of adenohypophyseal hormones are generated by distinct amplitude and/or frequency modulation of underlying pituitary secretory bursts. J. Clin. Endocrinol. Metab. 71:1616-1623.

36. Spiegel, K., M. Follenius, J. Krieger, E. Sforza, and G. Brandenberger. 1995. Prolactin secretion during sleep in obstructive sleep apnea patients. $J$. Sleep. Res. 4:56-62.

37. Bradley, E.M., and C. Waterhouse. 1966. Effect of estrogen administration on extravascular cortisol. J. Clin. Endocrinol. Metab. 26:705-714.

38. Van Cauter, E., E.T. Shapiro, H. Tillil, and K.S. Polonsky. 1992. Circadian modulation of glucose and insulin responses to meals: relationship to cortisol rhythm. Am. J. Physiol. 262:E467-E475.

39. Yamada, Y., J. Yamamoto, A. Fujiki, Y. Hishikawa, and Z. Kaneko. 1967. Effect of butyrolactone and gamma-hydroxybutyrate on the EEG and sleep cycle in man. Electroencephalogr. Clin. Neurophysiol. 22:558-562.

40. Gronfier, C., R. Luthringer, M. Follenius, N. Schaltenbrand, J.P. Macher, A. Muzet, and G. Brandenberger. 1996. A quantitative evaluation of the relationships between growth hormone secretion and delta wave electroencephalographic activity during normal sleep and after enrichment in delta waves. Sleep (Rochester). 19:817-824

41. Krueger, J., and F.J. Obal. 1993. Growth hormone-releasing hormone and interleukin-1 in sleep regulation. FASEB J. 7:645-652.

42. Takahara, J., S. Yunoki, W. Yakushiji, J. Yamauchi, Y. Yamane, and T. Ofuji. 1977. Stimulatory effects of gamma-hydroxybutyric acid on growth hormone and prolactin release in humans. J. Clin. Endocrinol. Metab. 44:10141017.

43. Scrima, L., P.G. Hartman, F.H. Johnson, E.F. Thomas, and F.C. Hiller. 1990. The effects of gamma-hydroxybutyrate on the sleep of narcolepsy patients: a double blind study. Sleep (Rochester). 13:479-490.
44. Gerra, G., R. Caccavari, B. Fontanesi, A. Marcato, G. Fertonani Affini, D. Maestri, P. Avanzini, R. Lecchini, R. Delsignore, and A. Mutti. 1994. Flumazenil effects on growth hormone response to gamma-hydroxybutyric acid. Int. J. Clin. Psychopharmacol. 9:211-215.

45. Gallimberti, L., M. Cibin, P. Pagnin, R. Sabbion, P.P. Pani, R. Pirasta, S.D. Ferrara, and G.L. Gessa. 1993. Gamma-hydroxybutyric acid for treatment of opiate withdrawal syndrome. Neuropsychopharmacology. 9:77-81.

46. Dyer, J.E. 1991. Gamma-hydroxybutyrate: a health-food producing coma and seizure-like activity. Am. J. Emerg. Med. 1991:321-324.

47. Chin, M.Y., R.A. Kreutzer, and J.E. Dyer. 1992. Acute poisoning from gamma-hydroxybutyrate in California. West. J. Med. 156:380-384.

48. Steele, M.T., and W.A. Watson. 1995. Acute poisoning from gammahydroxybutyrate (GHB). MO. Med. 92:354-357.

49. Ross, T.M. 1995. Gamma-hydroxybutyrate overdose: two cases illustrate the unique aspects of this dangerous recreational drug. J. Emerg. Nurs. 21: 374-376.

50. Friedman, J., R. Westlake, and M. Furman. 1996. "Grievous bodily harm": Gamma-hydroxybutyrate abuse leading to a Wernicke-Korsafoff syndrome. Neurology. 46:469-471.

51. Spiegel, K., R. Luthringer, M. Follenius, N. Schaltenbrand, J.P. Macher A. Muzet, and G. Brandenberger. 1995. Temporal relationship between prolactin secretion and slow-wave electroencephalographic activity during sleep Sleep (Rochester). 18:543-548.

52. Rudman, D., A.G. Feller, H.S. Nagraj, G.A. Gergans, P.Y. Lalitha, A.F. Goldberg, R.A. Schlenker, L. Cohn, Y.W. Rudman, and D.E. Mattson. 1990 Effects of human growth hormone in men over 60 years old. N. Engl. J. Med. 323:1-6.

53. Rudman, D., A.G. Feller, L. Cohn, K.R. Shetty, I.W. Rudman, and M.W. Draper. 1991. Effects of human growth hormone on body composition in elderly men. Horm. Res. (Basel). 36(Suppl. 1):73-81.

54. Marcus, R., G. Butterfield, L. Holloway, L. Gilliland, D.J. Baylink, R.L. Hinz, and B.M. Sherman. 1990. Effects of short term administration of recombinant human growth hormone to elderly people. J. Clin. Endocrinol. Metab. 70:519-527.

55. Papadakis, M.A., D. Grady, and D. Black. 1996. Growth hormone replacement in healthy older men improves body composition but not functional ability. Ann. Intern. Med. 124:708-716.

56. Bowers, C.Y. 1994. On a peptidomimetic growth hormone-releasing peptide. J. Clin. Endocrinol. Metab. 79:940-942.

57. Saenger, P. 1996. Oral growth hormone secretagogues-better than Alice in Wonderland's growth elixir? J. Clin. Endocrinol. Metab. 81:2773-2775.

58. Chapman, I.A., M.A. Bach, E. Van Cauter, M. Farmer, D. Krupa, A.M Taylor, L.M. Schilling, K.Y. Cole, E.H. Skyles, S.S. Pezzoli, et al. 1996. Stimulation of the growth hormone (GH)-insulin-like growth factor-I axis by daily oral administration of a GH secretagogue (MK-677) in healthy elderly subjects. $J$. Clin. Endocrinol. Metab. 81:4249-4257. 